\title{
Left Atrial Myxoma in a Child: An Uncommon Presentation of a Rare Tumour in Early Age
}

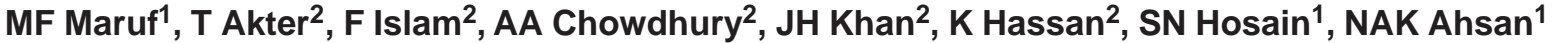 \\ ${ }^{1}$ Deptt. Of Cardiothoracic Surgery, Chittagong Medical College Hospital, \\ ${ }^{2}$ Deptt. of Cardiovascular Surgery, NICVD
}

\begin{abstract}
Keywords: $\quad$ Myxomas are rare tumours but are the most common benign tumours of the heart. They can arise Myxoma, Left Atrium from any heart chamber. However, they arise more frequently from the left atrium. They have rarely been described as originating in early age. A case of left atrial myxoma successfully removed using cardiopulmonary bypass in a 8-year-old child is presented. Review of the literature emphasizes the rarity and clinically aggressive behavior of this tumor in this age group. The object of this case report is to present myxoma in children and to evaluate possible differences between young and adult patients.
\end{abstract}

(Cardiovasc. j. 2012; 4(2): 171-173)

\section{Introduction:}

Primary heart tumors are uncommon in patients of pediatric age, 1 with a reported incidence of $0.2 \%$ in children referred for cardiac disease, ${ }^{2}$ whereas only $14.2 \%$ of all cardiac tumors occur in patients aged less than 16 years. $\stackrel{3}{ }$ Although cardiac myxoma is by far the most common primary heart tumor in adults,,$\frac{4.5}{r h a b d o m y o m a ~ i s ~ t h e ~ m o s t ~ f r e q u e n t l y ~}$ encountered heart tumor in infancy and childhood. ${ }^{2,6-11}$ We report on our experience with surgical treatment of primary cardiac tumors in childhood.

Primary cardiac tumors are rare, with an estimated incidence of $0.05 \%$ in postmortem series. Of these, atrial myxomas are responsible for up to $50 \%$ of cases. However, they are rare in teenagers and are extremely uncommon in preschool children. We report surgical removal of a left atrial myxoma in an 8-year-old child.

\section{Case report:}

A 8-year-old girl had presented on several occasions to her general practitioner over a 6month period with cough, increasing breathlessness, "flu-like" symptoms, anorexia, and weight loss. Treatment with bronchodilators and antibiotics had been successful in alleviating symptoms.
A sudden worsening of her symptoms and the onset of peripheral edema and ascites led to emergency admission to the peripheral hospital, where chest radiography revealed gross cardiomegaly and pulmonary edema. Then she was admitted in NICVD.

On arrival, she was tachypneic and tachycardic with her liver palpable at $6 \mathrm{~cm}$ below the costal margin. The apex beat was displaced to the midaxillary line, and a right ventricular heave was readily palpable. Her jugular venous pressure was elevated to her ears, and auscultation revealed a prominent pulmonary component to her second heart sound and a pansystolic murmur of mitral regurgitation. There was evidence of crepitations in both lungs. She was treated with anti failure drugs.

Echocardiography revealed a large, homogeneous mass arising from the inter atrial septum and prolapsing into the left ventricle. The mass was seen to be almost completely obstructing the mitral orifice during diastole and occupying the entire atrium during systole .Gross mitral regurgitation was evident. After initial recovery from her heart failure she was The patient was then referred to Department of Cardiovascular and Thoracic surgery and was planned for excision of LA Myxoma.

Address of Correspondence: Dr Mohammad Fazle Maruf, Deptt. of Cardiothoracic surgery,Chittagong Medical College, Chittagong, Bangladesh. 
Following sternotomy, routine cardiopulmonary bypass (CPB) was established through aortic and bi-caval cannulation. The heart was arrested using cold blood cardioplegia.

After cardioplegic arrest, the left atrium was opened. A large myxoma with its pedicle attached with inters atrial septum distorting the mitral valve and prolapsing into the left ventricle was found. The tumor together with the entire pedicle and its base in septum was excised keeping the septum intact. The mitral valve was found to be undamaged and the annulus not dilated. The rest of the heart was inspected for further tumors, the left atrial wall was closed directly, and the patient weaned from bypass uneventfully.

Over the next week, the child made an uneventful recovery with improved mitral valve function on echocardiography. Histological examination revealed the mass to be an atrial myxoma, with complete excision of the base.

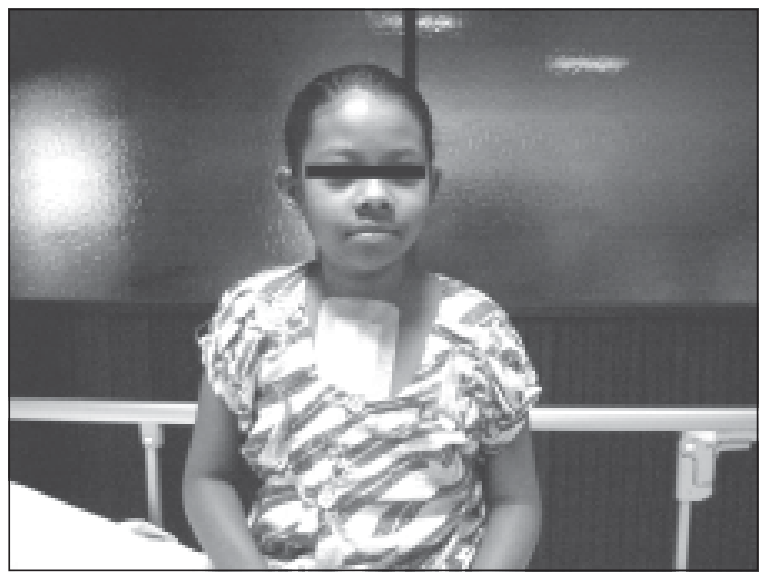

Fig-1: 8 yrs girl having LA Myxoma (post operative picture)

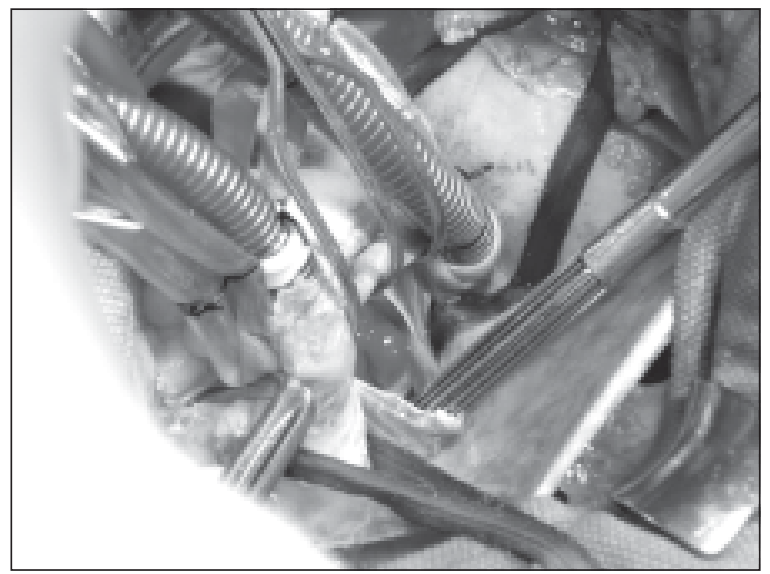

Fig-2: LA Myxoma Attached with inter atrial septum with pedicle (arrow).

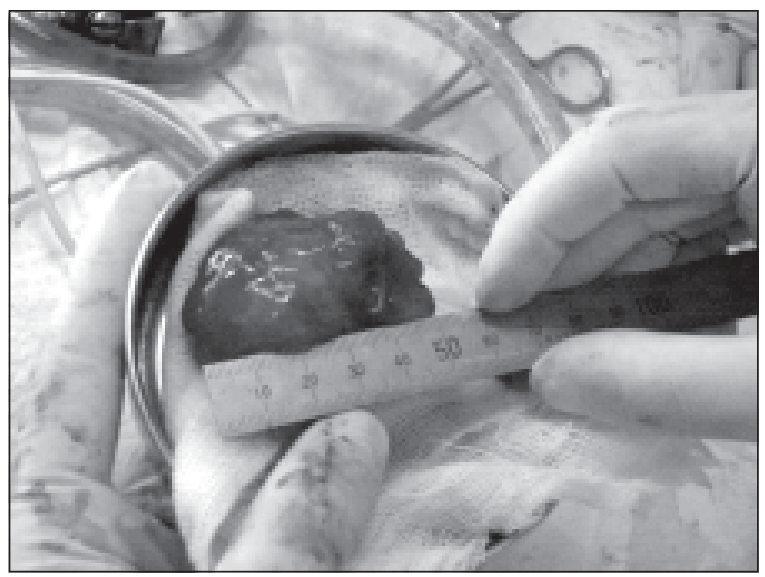

Fig-3: LA myxoma, just after excision

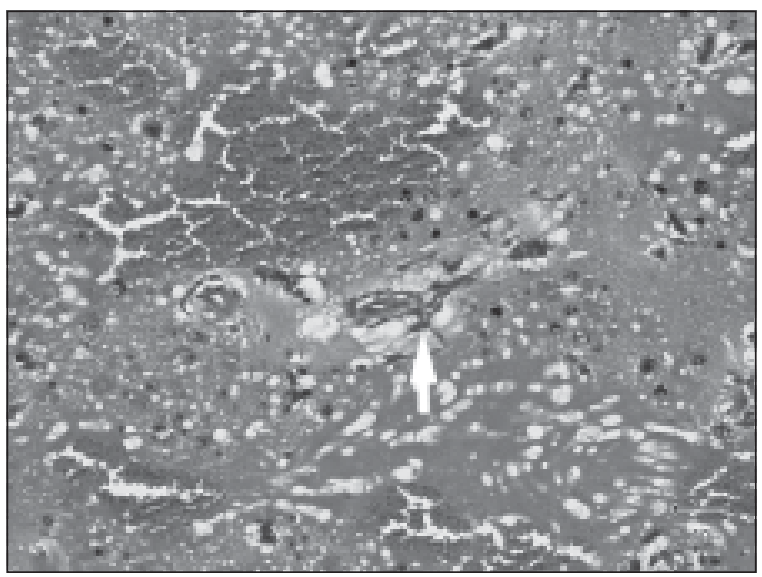

Fig-4: Histological study of atrial myxoma.

\section{Discussion:}

The clinical manifestations can be divided into a triad of symptoms: obstructive, embolic or constitutional (Goodwin's triad). $\underline{2}, \underline{5}$ Two of the classic triad of symptoms commonly found in this condition was present in our case. First, obstruction to the outflow of the cardiac chambers, leading to intermittent cardiac failure, was present for at least 6 months. This is the most commonly reported symptom and could account for the postural dependence of syncope experienced in this group. Second, our patient experienced vague symptoms of malaise, anorexia, weight loss, and a "flu-like syndrome" also common in this condition. Fortunately, embolism, which can also occur in up to $50 \%$ of these children and have devastating sequelae, was not a feature.

Echocardiography provides an accurate, noninvasive method of diagnosing these intracardiac masses at the bedside. The addition 
of transesophageal probes for use in the pediatric population adds further useful information regarding the site of attachment of these tumors and makes differentiation from thrombus more reliable. Although these tumors are histologically benign and not known to invade beyond the subendocardial layers, anecdotal reports of "malignant behavior" in these tumors mandate fullthickness excision of the base of the tumor and surrounding myocardium. The usual origin of the stalk of the tumor in our patient from the inter atrial septum makes it easy to define the extent of endocardial resection, because recurrences have been reported to occur more commonly in "extraseptal" myxomas. 1

In contrast to the situation in adults, rhabdomyomas, fibromas, and teratomas are more common in children than myxomas. ${ }^{2}$

The first successful removal of a myxoma in an infant was in 1967. $\underline{3}$ With advances in extracorporeal technology, a complete cure can be expected in most of these cases, in contrast to the prebypass era. $\stackrel{4}{ }$ Hemodynamic collapse and systemic or pulmonary embolization from these masses are dangers during operations on these children.

The paucity of similar reports could be attributed to the rarity of the condition in this age group, together with the delays inherent in diagnosis. Due to the smaller hearts and the rapid rate of growth of most tissues in children, these tumors may prove fatal much earlier in the course of their natural history.
The treatment is surgical and should not be deferred even in asymptomatic patients because of the embolic tendency and haemodynamic instability mainly due to valvular compromise causing sudden death. $., \underline{1}, \underline{9}$

\section{References:}

1. Mac Gowan SW, Sidhy P, Aheme T, Luke D,Wood AE, Neligan MC. Atrial myxoma:National incidence diagnosis and surgical management. Isr J Med Sci 1993; $162: 223-26$

2. Arciniegas E, Hakimi M, Farooki ZQ, Truccone NJ, Green EW. Primary cardiac tumors in children. $J$ Thorac Cardiovasc Surg 1980;79:582-91.

3. Sanyal S, De Luchtenberg N, Rojas RH, Stansel HC, Browne MJ. Right atrial myxoma in infancy and childhood. Am J Cardiol 1967;20:263-9.

4. Goldberg HP, Glenn F, Dotter CT, Steinberg I. Myxoma of the left atrium: diagnosis made during life with operative and post mortem findings. Circulation 1952;6:762-7.

5. Simcha A, Wells BG, Tynan MT and Waterston DJ. Primary cardiac tumours in childhood. Arch Dis Child 1971;46:508-14.

6. Daubenton JD, Human DG, Hassoulas J, Mills AE. Left atrial myxoma in a 13-month old infant. South Afr Med J 1985;68:261-2.

7. Park JM, Garcia RR, Patrick JK, Wagner D, Anuras S. Right atrial myxoma with nonembolic intestinal manifestation. Pediatr Cardiol 1990;11:164-6.

8. Pasaoglu I, Demircin M, Ozkutlu S, Bozer AY. Right atrial myxoma in an infant. Jpn Heart J 1991;32:263-6.

9. Peters MN, Hall RJ, Cooley DA, et al. The clinical syndrome of atrial myxoma. JAMA 1974; 230:695701. 